\title{
A multiattribute utility evaluation for the detection of Cryptosporidium species in stool
}

\author{
Prem Singh Grover ${ }^{1}$, Rajesh Bareja ${ }^{2},{\text { Beena } \mathrm{Jad}^{3}}^{3}$ \\ ${ }^{1,2,3}$ (Department of Microbiology, M.M.I.M.S.R, Mullana, Ambala, Haryana, India - 133207)
}

\begin{abstract}
A total of 500 stool samples were subjected to examination with the help of direct microscopy and concentration methods. Of these, fifty two (10.4\%) specimens were positive for Cryptosporidium parvum. Among direct microscopy methods - direct wet mount, negative staining and modified Ziehl-Neelsen staining showed a positivity of $22(4.4 \%), 14(2.8 \%)$ and $39(7.8 \%)$ respectively. After the Formol-ether Concentration (FEC) technique, wet mount, negative staining and modified Ziehl-Neelsen stain were positive in 32 (6.4\%), 37 (7.4\%) and $52(10.4 \%)$ cases respectively. Wet mount after Saturated Sodium Chloride Salt Flotation (SSSF) technique was positive in only 6 (1.2\%) cases. Modified Ziehl-Neelsen stain after SSSF technique was positive in $19(3.8 \%)$ cases while negative staining after SSSF technique was positive in only 1 (0.2\%) case. Cryptosporidium parvum positivity was 1.18 times higher in males as compared to females. The results indicate that the modified Ziehl-Neelsen staining after FEC technique to detect Cryptosporidium parvum in the stool specimens has significantly higher accuracy $(p<0.001)$ and reliability as compared to all the other techniques.

Keywords: Cryptosporidium parvum; modified Ziehl-Neelsen stain; FEC; SSSF
\end{abstract}

\section{Introduction}

The most common clinical feature of cryptosporidiosis in immunocompetent as well as immunocompromised persons is diarrhea [1]. Cryptosporidium parvum (C. parvum) is associated with massive diarrhea outbreaks worldwide, generally caused by exposure to drinking or recreational water or direct contact with infected persons through the oral-fecal route [2,3]. Diarrhea is exceedingly common and exerts an enormous toll in terms of mortality, morbidity, loss of work productivity, consumption of medical resources and social inconvenience. In man, the incidence of Cryptosporidium is more in children. Stool is usually watery, greenish in colour containing mucus and vomiting is commonly associated. Other symptoms may be colic by abdominal pain, anorexia, nausea and abdominal distension. Frequencies of passing stool usually 3-6 times in immunocompromised patients but may be up to 20 times a day [1].

Incidence in India is reported to vary from 1.3\% to 13\% [4]. There are studies from India, which entail a number of diagnostic methods without any uniform pattern. The detection of Cryptosporidium in India is limited to major research laboratories and does not feature in protocols for routine investigations in most of the clinical laboratories. The complex lifecycle of Cryptosporidium species, its small size, and subtle staining characteristics have contributed to the problem for identification of this parasite in routine stool preparations [57].

The purpose of the present study was to evaluate two methods, taking into account several attributes of diagnostic testing. The attributes of each of the tests evaluated were diagnostic yield, cost of performing the test, ease of handling, and ability to process large numbers of specimens for screening purposes by batching. A diagnosis of cryptosporidiosis may have direct benefits to the patient and physician by providing a clinical diagnosis and limiting extensive diagnostic evaluations. It may also reduce the use of empirical therapy for gastroenteritis, which could be ineffective and potentially harmful.

\section{Material And Methods}

The study was carried out in a tertiary care hospital over a period of twenty-four months. During this period, 500 stool samples received in the department of Microbiology for routine investigation were subjected to the study. The study included the patients of all age groups attending the OPD and having the history of diarrhea admitted in the hospital. After doing the macroscopic examination, direct microscopic examination was done. All the specimens were subjected to wet saline and iodine preparation $[8,9]$. Two smears were prepared directly from the specimen and stained with two different stains, modified Ziehl-Neelsen staining (MZN) by using 5\% sulphuric acid as a decolourizer and negative staining with carbol fuchsine (NS) respectively [8,9]. All the specimens were then subjected to two different concentration techniques, Formol-ether Concentration (FEC) and Saturated Sodium Chloride Salt Flotation Method (SSSF) [8,9]. Two smears each thus prepared after concentration method were subjected to staining with MZN and NS respectively. MZN smears and NS smears were observed under oil immersion and high power of bright-field microscope respectively. 


\section{Results}

Five hundred stool samples were subjected to parasitological examination. Of these, fifty two (10.4\%) specimens were positive for $C$. parvum (Fig.1). Among direct microscopy methods - direct wet mount, MZN and NS showed a positivity of $22(4.4 \%), 39(7.8 \%)$ and $14(2.8 \%)$ respectively. After the FEC technique (Fig. 2), wet mount, MZN and NS were positive in $32(6.4 \%), 52(10.4 \%)$ and $37(7.4 \%)$ cases respectively. Wet mount after SSSF technique was positive in only $6(1.2 \%)$ cases. MZN after SSSF technique was positive in 19 (3.8\%) cases while NS after SSSF technique was positive in only $1(0.2 \%)$ case (Table 1). C. parvum positivity was 1.18 times higher in males as compared to females (Table 2). Almost two-third positive cases were aged upto 30 years. Maximum incidence of positive cases (21 i.e. $40.38 \%$ ) was during months of June, July and August.

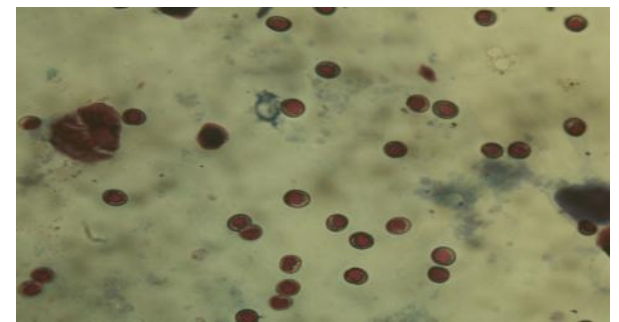

Fig. 1; oocysts of $C$. parvum in modified Z N stain preparation under oil immersion (100X)

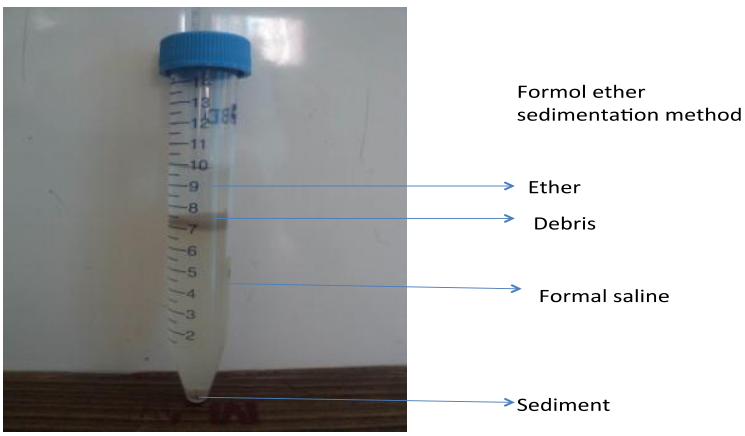

Fig. 2; formol-ether sedimentation technique for stool specimens

Table 1; microscopic examination of stool samples for C. parvum before and after concentration methods

\begin{tabular}{|c|c|c|c|}
\hline Total $(\mathrm{n}=500)$ & Direct Microscopy & $\begin{array}{c}\text { Microscopy after } \\
\text { Concentration method } \\
\text { (FEC) }\end{array}$ & $\begin{array}{c}\text { Microscopy after } \\
\text { Concentration method } \\
\text { (SSSF) }\end{array}$ \\
\hline Wet Mount & $22(4.4 \%)$ & $32(6.4 \%)$ & $6(1.2 \%)$ \\
\hline $\begin{array}{c}\text { Modified } \\
\text { Z N Staining }\end{array}$ & $39(7.8 \%)$ & $52(10.4 \%)$ & $19(3.8 \%)$ \\
\hline Negative Staining & $14(2.8 \%)$ & $37(7.4 \%)$ & $1(0.2 \%)$ \\
\hline
\end{tabular}

Table 2; Gender wise distribution of cases of C. parvum

\begin{tabular}{|c|c|c|c|c|}
\hline Sex & $\begin{array}{c}\text { No. of suspects } \\
(\mathrm{n}=500)\end{array}$ & $\begin{array}{c}\text { Positive no. of } \\
\text { cases }(\mathrm{n}=52)\end{array}$ & $\begin{array}{c}\% \text { (Out of total in } \\
\text { corresponding gender) }\end{array}$ & $\begin{array}{c}\% \text { (Out of total } \\
\text { positive) }\end{array}$ \\
\hline $\mathrm{M}$ & 288 & 32 & 11.1 & 61.5 \\
\hline $\mathrm{F}$ & 212 & 20 & 9.4 & 38.5 \\
\hline
\end{tabular}

\section{Discussion}

There is difficulty in the identification of C. parvum in stool. In view of this Centre for Disease Control and Prevention (CDC) in its guidelines has also recommended testing of multiple stool specimens before reporting a test to be negative. Considering high incidence of false negativity, a number of attempts had been made to modify, alter and upgrade the existing laboratory procedures for identification of $C$. parvum in human faecal specimen. In the present study, detection of $C$. parvum oocytes was done with the help of direct microscopy and concentrated stool methods. In concentrated stool assessment, two techniques, formol-ether sedimentation technique and saturated sodium chloride floatation technique were employed. All the assessments 
were done in three replicates, one each for wet mount, modified Ziehl-Neelsen stain and negative stain respectively.

In the present study, overall prevalence of $C$. parvum in stool specimen tested was $10.4 \%$. C. parvum is an important cause of diarrhoea with prevalence rates varying from 1 to $4 \%$ in the developed world and 6 to $19 \%$ in the developing world as shown in Table 3 [10-15].

Table 3; prevalence of $C$. parvum in different studies

\begin{tabular}{|c|c|c|c|c|}
\hline Sr. No. & Author & Place & Year of study & Prevalence \\
\hline 1. & Baxby and Hart $^{10}$ & UK & 1986 & $1.4 \%$ \\
\hline 2. & Sallon et al..$^{11}$ & Gaza & 1990 & $19 \%$ \\
\hline 3. & Kaur et al. $^{12}$ & Delhi & 2002 & $18.9 \%$ \\
\hline 4. & Das et al. ${ }^{13}$ & Kolkata & 2006 & $4.6 \%$ \\
\hline 5. & Nagamani et al. $^{14}$ & Secundrabad & 2007 & $7.6 \%$ \\
\hline 6. & Khurana et al. $^{15}$ & Chandigarh & 2012 & $6 \%$ \\
\hline 7. & Present study & Haryana & 2012 & $10.4 \%$ \\
\hline
\end{tabular}

Among direct microscopy methods - direct wet mount showed a positivity of 22/52 (42.3\%), direct staining examination by negative staining showed a positivity of $14 / 52(26.9 \%)$ while direct staining examination by MZN showed a positivity of 39/52 (75\%). Although some workers have reported relatively better efficacy of negative staining method but under electron microscopy [16,17]. Negative staining under light microscopy is recommended for capsular structures, as they appear clear in dark background [18]. Some authors have advocated the use of negative staining for identification and isolation of $C$. parvum species. They have also illustrated that the quality of negative staining is time dependent and if the samples are not viewed within a specified time then the spores might become less visible [15]. Moreover, the technique requires higher level of technical and optical skill while using the microscope and owing to this the results might vary among persons with different experience and microscopic skills.

Between two concentration techniques, formol-ether sedimentation technique showed relatively better results for all the three methods viz. wet mount, modified Ziehl-Neelsen staining and negative staining as compared to saturated sodium floatation technique. Best results were obtained for modified Ziehl-Neelsen staining under formol-ether sedimentation technique, which had a $100 \%$ positivity rate. For saturated sodium chloride floatation technique the results were poor for all the three methods (wet mount, MZN and NS) even when compared to direct staining methods. Casemore et al. (1985) have described formol-ether sedimentation method as the confirmatory method for identification of $C$. parvum and results in present study are in accordance with their observations. Kuzehkanan et al. (2011) have also found that formol-ether sedimentation method using Ziehl-Neelson stain gives precise results, which are comparable to molecular techniques such as PCR. However, Kar et al. (2011) have reported sodium chloride floatation technique using Ziehl-Neelsen stain to be superior in their ability to reduce the proportion of degenerated or non-viable oocysts. In the present study, the sodium chloride floatation technique showed results poorer than the direct microscopy and the reason for that could be attributed to the fact that sodium chloride floatation technique is not directed towards preservation of the oocytes and during the laboratory procedure there is possibility of its degeneration and hence poor positivity whereas, in formol-ether sedimentation, use of formalin during the procedure preserves the oocysts and hence the possibility of isolation and visualization is higher. SSSF is a most commonly employed concentration method for the detection of helminthic ova in routine testing of stool samples. This is cheap and easy to perform and needs minimum skills. It is a time-tested method. There is hardly any laboratory in India that has not employed this method. So it has come to stay a technique always coupled with routine saline and iodine preparation. Keeping this in mind this method was included in this study inspite of the fact that certain reports have expressed doubts about its usefulness but there are equivocal reports too [1,20]. Among different techniques, negative staining after SSSF showed poorest results which could be attributed to lower density of oocytes after purification, as already stated above, negative staining is not a suitable technique under low density of microbes especially in the light microscopy.

Among specimen processed, majority were from males (57.6\%). Only 212 (42.4\%) samples comprised of females. This is in contrast with the observations made by Chang et al. (2006) who observed that functional gastrointestinal disorders were more common in females as compared to males [21,22]. The contrast in our study could be because of the difference in hospital healthcare seeking behaviour of Indian population wherein females are generally tended to seek hospital healthcare only for life-threatening problems and for minor ailments, they generally seek home remedies $[23,24]$.

In the present study, Table 2 shows the proportion of males was higher as compared to that of females both for positivity (11.1\% against $9.4 \%)$ as well as for proportion of positives $(61.5 \%$ against $38.5 \%)$. In cryptosporidiosis surveillance, the proportion of females was found to be 1.15 times higher in females as 
compared to males [25]. However, Pereira et al. (2002) have also shown that the risk of infection among male patients was 2.2 times higher as compared to that in females among children hospitalized for diarrhea in Brazil. Kimani et al. (2012) have observed the proportion of males to be 3.35 times higher as compared to that of females in a study conducted at Kenya. Higher risk to males in developing countries could be attributed to higher prevalence of manual jobs, unhygienic conditions of work and eating environment and gender wise differentiation of occupations, which involve higher proportion of males as compared to females thus enhancing the risk of males as compared to females.

As for as the age distribution was concerned among specimen tested positive, the proportion of those aged $\leq 10$ years and aged between 21-30 years was maximum (23.1\%). Almost two-third (63.5\%) of positive cases were from amongst those aged upto 30 years. Hlavsa et al. (2005) in their nationwide surveillance study on cryptosporidiosis reported that greater number of cases were observed in age group 1-9 and 30-39 years compared with other age groups. Prevalence of $C$. parvum has been reported to be more than ten times that of adult cases in paediatric cases with diarrhoea [29]. The higher rate of prevalence among paediatric age group might be attributed to the lack of maintenance of hygiene while younger age group is generally more active in life and spends a lot of their time outdoors and is more exposed to various risk factors such as unhygienic food, inability to maintain hygiene and tendency to experiment with a variety of food stuff which may be unhygienic in nature.

A high seasonal variability was observed in number of samples obtained during different months. It has been reported maximum during the rainy season because of the availability of favourable conditions for growth of microbes and possibility of opportunistic infections [30].

On comparing the different diagnostic methods for their relative accuracy (accuracy to predict, true positive and true negative cases precisely), direct wet mount examination had significantly lower diagnostic accuracy as compared to direct staining examination by MZN, staining examination by negative staining after FEC and staining examination by MZN after FEC. It has a significantly higher diagnostic accuracy as compared to staining examination by Negative staining after SSSF $(p<0.05)$. None of the other comparisons were significant statistically $(\mathrm{p}>0.05)$. Direct staining examination by MZN showed significantly higher accuracy as compared to direct wet mount $(\mathrm{p}<0.05)$, direct staining by negative staining $(\mathrm{p}<0.001)$, MZN after SSSF and negative staining after SSSF $(\mathrm{p}<0.05)$. It had significantly lower accuracy as compared to MZN after FEC only $(\mathrm{p}<0.001)$. None of the other comparisons were significant statistically. Direct staining by negative staining had significantly lower accuracy as compared to direct examination by MZN, wet mount after FEC, MZN after FEC and negative staining after FEC. It did not show a significant difference from MZN after SSSF and negative staining after SSSF.

Wet mount after FEC had significantly higher efficacy as compared to direct staining by negative staining and staining examination by negative staining after SSSF only. It had significantly lower accuracy as compared to MZN after FEC only ( $<0.001$ ). All the other comparisons did not yield a significant difference. Negative staining after FEC had significantly higher accuracy as compared to direct wet mount examination, direct staining by negative staining, MZN after SSSF and Negative staining after SSSF. It had a significantly lower accuracy as compared to MZN after FEC. MZN after SSSF had significantly higher accuracy as compared to negative staining after SSSF only $(\mathrm{p}=0.040)$. It had significantly lower accuracy as compared to direct staining by MZN, MZN after FEC and negative staining after FEC ( $<<0.05)$. All the other comparisons were not significant statistically. Staining examination by MZN after FEC had significantly higher accuracy as compared to all the other methods $(\mathrm{p}<0.001)$.

\section{Conclusions}

Despite the comparative efficacy of different techniques and relatively better performance of FECmodified Ziehl-Neelsen staining, it is essential to make a note that identification of $C$. parvum is a daunting task for a microbiologist with varying accuracy. In the present study, the criteria for evaluating performance of different methods was taken as evaluation of efficacy of each method against a positive finding in a given sample. FEC-MZN combination showed $100 \%$ positivity against all the other techniques and hence can be considered as the best technique for routine stool examination. It improves the positivity considerably and is simple to perform and less time consuming.

\section{Acknowledgements}

We are thankful to Dr. Rakesh Sehgal, Professor, Department of Parasitology, Post Graduate Institute of Medical Research, Chandigarh, India for providing tactical support. 


\section{References}

[1] W.L. Current, and L.S. Garcia, Cryptosporidiosis, Clin. Microbial Rev., 4, 1991, 325-358

[2] R. Fayer, Cryptosporidium: a water-borne zoonotic parasite, Vet. Parasitol., 126, 2004, $37-56$.

[3] E. M. Goncalves, A. J. da Silva, M. B. Eduardo, I. H. Uemura, I. N. Moura, V. L. Castilho, and C. E. Corbett, Multilocus genotyping of Cryptosporidium hominis associated with diarrhea outbreak in a day care unit in Sao Paulo, Clinics, 61, 2006, 119126.

[4] G. Nath, A. Choudhary, B.N. Shukla, and T.B. Singh, Significance of C. parvum in acute diarrhoea in north eastern India, J. Med. Microbiol.,48, 1999, 523-526.

[5] R. Bird, and M. Smith, Cryptosporidiosis in man: parasite life cycle and fine structural pathology, J.Pathol., 132, 1980, 217-233.

[6] W. Current, Human cryptosporidiosis, N. Engl. J. Med., 309, 1983, 1325-1327.

[7] R. Fayer, and B. Ungar, Cryptosporidium spp. and cryptosporidiosis, Microbiol. Rev., 50, 1986, 458-483.

[8] K.D. Chatterjee, Parasitology, Protozoology and Helminthology (CBS Publishers and Distributers Pvt Ltd, New Delhi, India, 2009) 260-265.

[9] I. Garcia, and D.A. Bruckner, Diagnostic Medical Parasitology (ASM press, Washington, 1997) 608-649.

[10] D. Baxby, and C.A. Hart, The incidence of cryptosporidiosis: a two year prospective survey in children's hospital, J. Hygiene., 96, 1986, 107-111.

[11] S. Salon, R.E. Showwa, M.E. Masri, M. Khalil, N. Blundell, and C.A. Hart, Cryptosporidiosis in children in Gaza, Ann. Trop. Paediatr., 11, 1990, 277-281.

[12] R. Kaur, D. Rawat, M. Kakkar, B. Uppal, and V.K. Sharma, Intestinal parasite in children with diarrhea in Delhi, India, Sothern Asian J. Trop. Med. Public Health, 33, 2002, 725-728.

[13] P. Das, S.S. Roy, K. MitraDhar, P. Dutta, M.K. Bhattacharya, and A. Sen, Molecular characterization of Cryptosporidium spp. from children in Kolkata, India, J. Clin. Microbiol., 44, 2006, 4246-4249.

[14] K. Nagamani, P.R. Pavuluri, M. Gyaneshwari, K. Prasanthi, M.I. Rao, and N.K. Saxena, Molecular characterization of Cryptosporidium: An emerging parasite, Indian J. Med. Microbiol., 25, 2007, 133-6

[15] S. Khurana, P. Sharma, A. Sharma, and N. Malla, Evaluation of Ziehl-Neelsen staining, auramine phenol staining, antigen detection enzyme linked immunosorbent assay and polymerase chain reaction, for the diagnosis of intestinal Cryptosporidiosis, Tropical Parasitology, 2, 2012, 20-23

[16] D.P. Casemore, M. Armstrong, and R.L. Sands, Laboratory diagnosis of cryptosporidiosis, J. Clin. Pathol., 38, 1985, 1337-1341

[17] D. Baxby, N. Blundell, and C.A. Hart, The development and performance of a simple, sensitive method for the detection of Cryptosporidium parvum oocysts in faeces, J. Hyg. Camb., 92, 1984, 317-323

[18] J.G. Black, Microbiology Principles and Explorations (John Wiley \& Sons, 2004).

[19] A.B. Kuzehkanan, M. Rezaeian, and H. Zeraati, A sensitive and specific PCR based method for identification of Cryptosporidium parvum spp. using new primers from 18S Ribosomal RNA. Iranian J. Parasitol., 6(4), 2011, 1-7.

[20] S. Kar, S. Gawlowska, A. Daugschies, and B. BangouraB, Quantitative comparison of different purification and detection methods for Cryptosporidium parvum oocysts, Vet. Parasitol., 177(3-4), 2011 May, 366-70.

[21] L. Chang, B.B. Toner, S. Fukudo et al., Gender, Age, Society, Culture, and the patient's perspective in the functional gastrointestinal disorders, Gastroenterology, 130, 2006, 1435-1446.

[22] S.L.S. Halder, G.R. Lokie III, and C.D. Schleck, Natural history of functional gastrointestinal disorders: A 12-year longitudinal population-based study, Gastroenterology, 133, 2007, 799-807.

[23] P. Bentley, and A. Parekh, Perceptions of anemia and health-seeking behavior among women in four Indian states. Technical Working Paper \#9, October 1998, The office of health and nutrition, bureau for global programs, field support and research, U.S. Agency for International Development.

[24] J. Sen, A sociological study on health seeking behavior of women with reference to hepatitis and gall bladder stone in south Assam, Ethno. Med., 6(2), 2012, 131-139.

[25] J.S. Yoder, R.M. Wallace, S.A. Collier, M.J. Beach, and M.C. Hlavsa, Cryptosporidiosis Surveillance - United States, $2009-2010$. Surveillance Summaries, 61(SS05), 2012, 1-12.

[26] M.D. Pereira, E.R. Atwill, A.P. Barbosa, S.A. Silva , and M.T. Garcia-Zapata, Intra-familial and extra-familial risk factors associated with Cryptosporidium parvum infection among children hospitalized for diarrhea in Goiânia, Goiás, Brazil, Am. J. Trop. Med. Hyg., 66(6), 2002, 787-793.

[27] V.N. Kimani, G. Mitoko, B. McDermott et al., Social and gender determinants of risk of cryptosporidiosis, an emerging zoonosis, in Dagoretti, Nairobi, Kenya. Tropical Animal Health and Production, 44(1), 2012, 17-23.

[28] M.C. Hlasva, J.C. Watson, M.J. Beach, Cryptosporidiosis Surveillance - United States 1999-2002. CDC MMWR Surveillance Summaries, 54(SSO1), 2005, 1-8.

[29] F.F. Natividad, C.C Buerano, C.B. Lago, C.A. Mapua, B.B. de Guzman, E.B. Seraspe, L.P. Samentar, and T. Endo, Prevalence rates of Giardia and Cryptosporidium parvum among diarrheic patients in the Philippines, Southeast Asian J. Trop. Med. Public Health., 39(6), 2008, 991-999.

[30] P.A. Prieto, R.L. Finley, P.K. Muchal et al., Burden of self-reported acute gastrointestinal illness in Cuba, J. Health Popul. Nutr. 27(3), 2009, 345-357. 